\title{
Transient resolution of venous sinus stenosis after high-volume lumbar puncture in a patient with idiopathic intracranial hypertension
}

\author{
Thomas J. Buell, MD, ${ }^{1}$ Daniel M. S. Raper, MBBS, ${ }^{1}$ I. Jonathan Pomeraniec, MD, MBA, ${ }^{1}$ \\ Dale Ding, MD, ${ }^{1}$ Ching-Jen Chen, MD, ${ }^{1}$ Davis G. Taylor, MD, ${ }^{1}$ and Kenneth C. Liu, MD1,2 \\ Departments of ${ }^{1}$ Neurosurgery and ${ }^{2}$ Radiology, University of Virginia Health System, Charlottesville, Virginia
}

\begin{abstract}
Stenosis of the transverse sinus (TS) and sigmoid sinus (SS), with a trans-stenosis pressure gradient, has been implicated in the pathophysiology of idiopathic intracranial hypertension (IIH). MRI has shown improvement in TS and SS stenosis after high-volume lumbar puncture (HVLP) in a subset of patients with IIH. The authors present the first report of an IIH patient with immediate post-HVLP TS and SS trans-stenosis pressure gradient reduction and an attendant increase in TS and SS cross-sectional area confirmed using intravascular ultrasonography (IVUS). Recurrence of the patient's TS-SS stenosis coincided with elevated HVLP opening pressure, and venous sinus stent placement resulted in clinical improvement. This report suggests that TS and SS stenosis may be a downstream effect of elevated intracranial pressure in $\mathrm{IH}$, rather than its principal etiological mechanism. However, the authors hypothesize that endovascular stenting may obliterate a positive feedback loop involving trans-stenosis pressure gradients, and still benefit appropriately selected patients.
\end{abstract}

https://thejns.org/doi/abs/10.3171/2017.3.JNS163181

KEY WORDS idiopathic intracranial hypertension; stenosis; intracranial venous sinus; intravascular ultrasonography; venous manometry; diagnostic technique

$\mathrm{T}$ RANSVERSE sinus (TS) and sigmoid sinus (SS) stenosis, with a trans-stenosis pressure gradient, has been implicated in the pathophysiology of idiopathic intracranial hypertension (IIH). ${ }^{2}$ TS and SS stenosis may cause venous outflow obstruction, reduction in CSF bulk flow, and elevated intracranial pressure (ICP). However, it is incompletely understood whether TS and SS stenosis is actually a downstream effect of $\mathrm{IIH}$, rather than its principal etiological mechanism. ${ }^{1}$ Although no definitive conclusion may be drawn from a single report, we provide evidence in support of the former. In this report, we document immediate resolution of TS and SS stenosis in a patient with IIH after ICP reduction using high-volume lumbar puncture (HVLP). We are the first to use catheter venous manometry simultaneously with intravascular ultrasonography (IVUS) to study intracranial venous occlusive disease.

\section{Case Report}

History and Examination

A 32-year-old, severely obese woman (body mass index [BMI] $51.6 \mathrm{~kg} / \mathrm{m}^{2}$ ) previously diagnosed with IIH presented to the University of Virginia Health System after failed medical management with acetazolamide. The patient reported severe headache and visual obscurations. A formal ophthalmological examination revealed bilateral papilledema. Brain MRI showed findings consistent with IIH, including an empty sella with slit ventricles, without structural lesions (Fig. 1A and B). Contrast MRI showed severe focal stenosis of the TS-SS junction bilaterally (Fig. 1C). The patient elected to proceed with diagnostic cerebral angiography and venous manometry. After 6-vessel catheter angiography showed no arterial abnormalities, venography was performed, venous pressures were recorded, and

ABBREVIATIONS BMI = body mass index; HVLP = high-volume lumbar puncture; ICP = intracranial pressure; IIH = idiopathic intracranial hypertension; IVUS = intravascular ultrasonography; MVP = mean venous pressure; $\mathrm{SS}$ = sigmoid sinus; $\mathrm{TS}$ = transverse sinus.

SUBMITTED December 19, 2016. ACCEPTED March 6, 2017.

INCLUDE WHEN CITING Published online August 25, 2017; DOI: 10.3171/2017.3.JNS163181. 

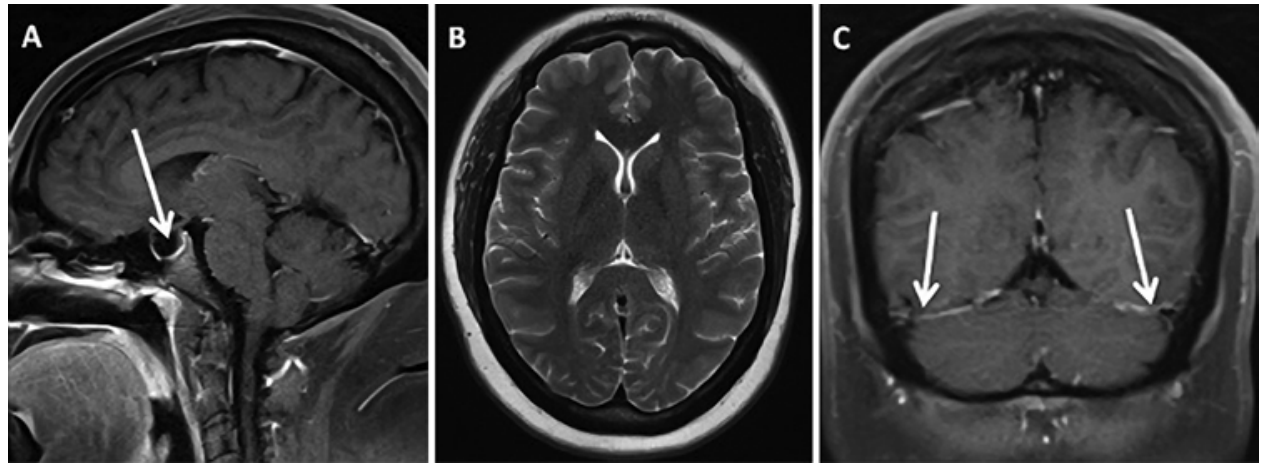

FIG. 1. A 32-year-old severely obese (BMI $51.6 \mathrm{~kg} / \mathrm{m}^{2}$ ) woman with IIH presented to the University of Virginia Health System after failed medical management with acetazolamide. The patient reported severe headache and visual obscurations. Formal ophthalmological examination revealed bilateral papilledema. Sagittal and axial brain MRI showed findings consistent with IIH, including an empty sella with slit ventricles, without structural lesions (A and B). Contrast-enhanced coronal MRI showed severe focal stenosis of the TS-SS junction bilaterally (C). Arrows indicate empty sella sign (A) and bilateral transverse sinus stenosis (C).

venous sinus cross-sectional areas were measured using IVUS (Volcano Corp.). Venography, with superior sagittal sinus injections in a lateral oblique view, showed severe TS and SS stenosis bilaterally, associated with a $38 \mathrm{~mm}$ $\mathrm{Hg}$ trans-stenosis pressure gradient and cross-sectional area of $12.9 \mathrm{~mm}^{2}$ (Fig. 2A and B).

\section{Operation}

A fluoroscopic HVLP was performed in the angiography suite without complication, and revealed an opening pressure of $52 \mathrm{~cm} \mathrm{H}_{2} \mathrm{O}$ that decreased to $23 \mathrm{~cm} \mathrm{H}_{2} \mathrm{O}$ after $32 \mathrm{ml}$ of normal CSF was removed. Immediate post-HVLP venography demonstrated radiographic improvement in bilateral TS and SS stenosis, corroborated by a reduction in the focal TS-SS trans-stenosis pressure gradient to $6 \mathrm{~mm} \mathrm{Hg}$, and an increase in the TS-SS junction cross-sectional area to $24.5 \mathrm{~mm}^{2}$ (Fig. 2C and D). Maximum mean venous pressure (MVP) recorded in the superior sagittal sinus decreased from $62 \mathrm{~mm} \mathrm{Hg}$ to 27 $\mathrm{mm} \mathrm{Hg}$. After the procedure, the patient's headaches and visual acuity improved, and the patient was discharged without further intervention.

\section{Postoperative Course}

The patient presented 1 month after discharge with recurrent symptoms. A repeat lumbar puncture revealed an elevated opening pressure of $33 \mathrm{~cm} \mathrm{H}_{2} \mathrm{O}$. Repeat venography demonstrated recurrent TS-SS junction stenosis bilaterally, now associated with a $26 \mathrm{~mm} \mathrm{Hg}$ trans-stenosis pressure gradient (Fig. 3A). We performed endovascular stenting of the right TS-SS junction using a single Zilver $8 \times 60-\mathrm{mm}$ self-expanding stent (Cook Medical), which reduced the trans-stenosis pressure gradient to $2 \mathrm{~mm} \mathrm{Hg}$ (Fig. 3B). Follow-up CT venography showed no recurrent stenosis of the stented venous sinus. Clinically, the patient reported continued improvement at last follow-up, 2 months after the stenting procedure.

\section{Discussion}

Prior studies used MRI to show improvement in venous sinus stenosis after HVLP in patients with $\mathrm{IIH},{ }^{3}$ but noninvasive measurements are prone to error from acquisi- tion artifact. We present the first report of a patient with IIH who underwent pre- and immediate post-HVLP diagnostic venography, catheter venous manometry (the gold standard for diagnosing venous stenosis), and intracranial dural sinus cross-sectional area measurement with IVUS. The patient's severe TS and SS stenosis resolved immediately after ICP reduction with HVLP. Venous manometry showed decreased TS-SS pressure gradients to less than 8 $\mathrm{mm} \mathrm{Hg}$, a criteria many authors use for stent placement, ${ }^{2}$ and there was a simultaneous increase in TS-SS junction cross-sectional area on IVUS imaging.

Treatment for patients with IIH often begins with conservative management such as weight loss, medications to reduce CSF production, and serial HVLP. ${ }^{5}$ Despite conservative therapy, many patients develop progressive symptoms or papilledema with visual decline. In medically refractory patients with IIH, more invasive treatments are available, such as lumboperitoneal shunting, ventriculoperitoneal shunting, or optic nerve sheath fenestration. ${ }^{8}$ More recently, a subset of patients with IIH and venous sinus stenosis has been reported to benefit from venous sinus stenting. ${ }^{2}$ In carefully selected cases, venous sinus stenting may even be an effective treatment for patients with symptomatic venous sinus stenosis without $\mathrm{IIH}{ }^{2,6}$

Our institution's venous sinus stenting protocol has been previously described. ${ }^{4,6,7}$ Briefly, patients are premedicated with $325 \mathrm{mg}$ of aspirin and $75 \mathrm{mg}$ of clopidogrel daily to achieve therapeutic ARU $(<551)$ and P2Y12 $(<208)$ levels. Patients are brought to the angiography suite, and general anesthesia is induced. We administer intravenous heparin according to a weight-based protocol and then obtain venous access. A triaxial shuttle is navigated into the internal jugular vein ipsilateral to the stenosis, which gives enough catheter support to navigate microguidewires into the venous sinuses. Then, under continuous fluoroscopic guidance, we deploy an appropriately sized stent across the target area of stenosis. Poststent pressure gradients are recorded and a final venogram is obtained. Patients typically are discharged the following day.

Currently, the pathophysiology of IIH is incompletely understood. Although stenosis of an intracranial dural sinus is implicated, it is unclear if this is the underlying 

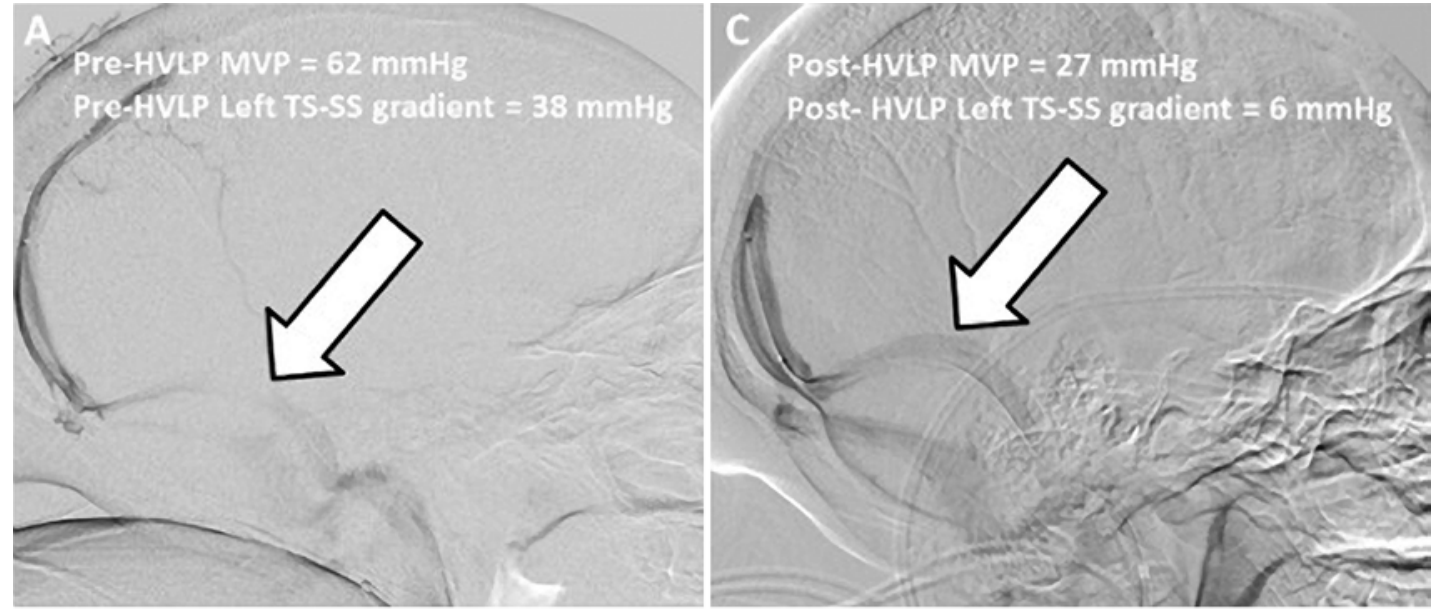

B
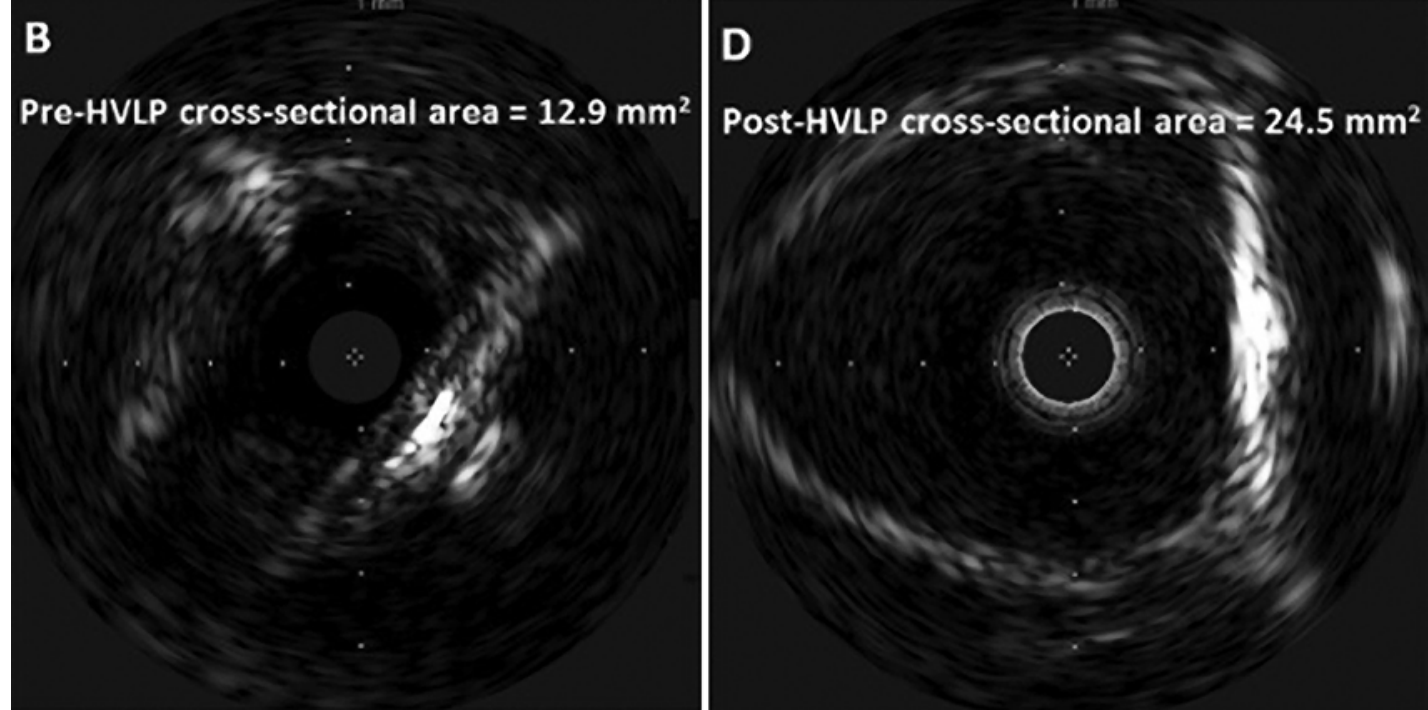

FIG. 2. Diagnostic venography, catheter venous manometry, and IVUS imaging were performed immediately before and after HVLP. Pre-HVLP venography (A) showed severe bilateral TS-SS stenosis, associated with a $38 \mathrm{~mm} \mathrm{Hg}$ trans-stenosis pressure gradient, an elevated MVP of $62 \mathrm{~mm} \mathrm{Hg}$, and IVUS imaging (B) cross-sectional area measuring $12.9 \mathrm{~mm}^{2}$. HVLP was performed without complication. The opening pressure was $52 \mathrm{~cm} \mathrm{H}_{2} \mathrm{O}$, which decreased to $23 \mathrm{~cm} \mathrm{H}_{2} \mathrm{O}$ after removing $32 \mathrm{ml}$ of normal CSF. Immediate post-HVLP venography (C) showed resolution of the prior TS-SS stenosis, now with a TS-SS trans-stenosis pressure gradient of $6 \mathrm{~mm} \mathrm{Hg}$, MVP of $27 \mathrm{~mm} \mathrm{Hg}$, and IVUS imaging (D) cross-sectional area measuring $24.5 \mathrm{~mm}^{2}$. Arrows indicate stenosis of the TS.

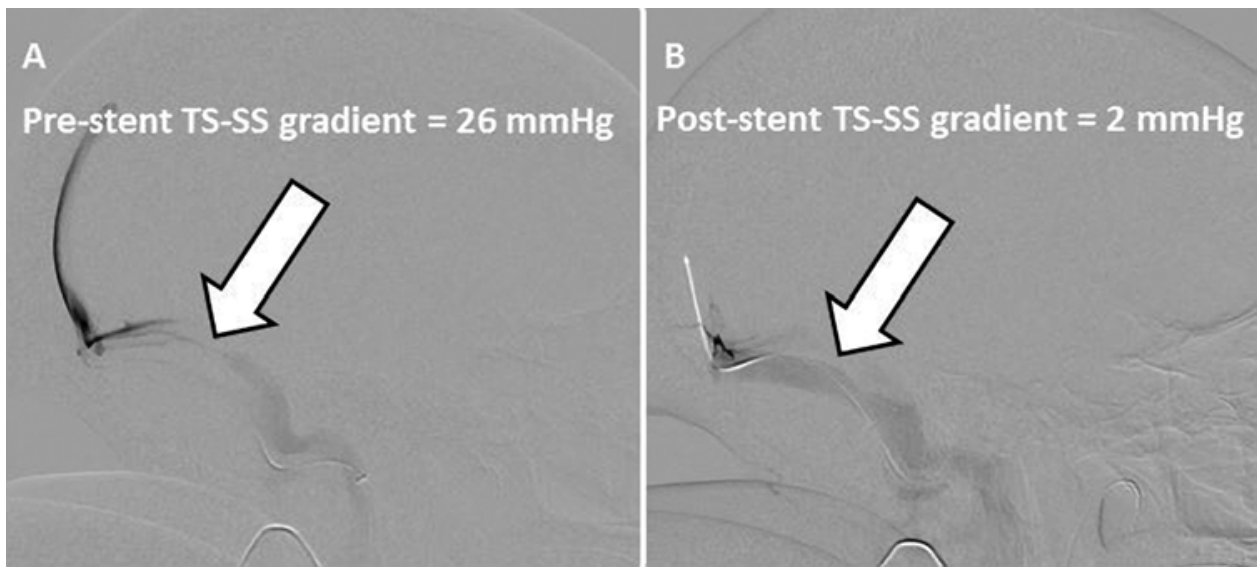

FIG. 3. Follow-up venography re-demonstrated severe TS-SS stenosis bilaterally, now associated with a $26 \mathrm{~mm} \mathrm{Hg}$ trans-stenosis pressure gradient $(\mathbf{A})$. We performed endovascular stenting of the right TS-SS junction using a single $8 \times 60-\mathrm{mm}$ self-expanding stent, which reduced the trans-stenosis pressure gradient to $2 \mathrm{~mm} \mathrm{Hg}(\mathbf{B})$. Arrows indicate stenosis of the TS. 
cause or the downstream effect of elevated ICP. ${ }^{1}$ Our report suggests the latter, because the patient's TS-SS junction stenosis resolved immediately after ICP reduction with HVLP, and recurred when ICP was elevated. If TS and SS stenosis is a result of elevated ICP, TS and SS stent placement may not be targeting the central pathophysiological mechanism of IIH. However, some authors have proposed a positive feedback loop mechanism in which venous sinus stenosis causes venous congestion, decreased CSF bulk flow, and elevated ICP, which ultimately compresses weak areas of the venous sinuses in a Starling resistorlike mechanism, further worsening the stenosis. ${ }^{1}$ If endovascular treatment with venous sinus stenting obliterates trans-stenosis pressure gradients, this positive feedback loop may be interrupted. Therefore, appropriately selected patients with IIH, with venous sinus stenosis and elevated trans-stenosis pressure gradients, may ultimately benefit from endovascular stenting. Although definitive conclusions cannot be made from a single report, we hypothesize that disruption of this positive feedback loop may explain the clinical improvement observed in our patient. Further prospective clinical studies are necessary to test this hypothesis and identify appropriate patient selection criteria for venous sinus stenting in medically refractory IIH.

Unfortunately, we do not have a case in which permanent CSF diversion alleviated venous sinus stenosis. At our institution, patients with IIH and venous sinus stenosis typically undergo stent placement.,6,7 This institutional practice bias is a limitation of our report and other retrospective studies from our institution, and may be eliminated through a prospective randomized trial. We hope to undertake such a trial in the future.

TS and SS stenosis with a trans-stenosis pressure gradient has been implicated in IIH pathophysiology. MRI has shown improvement in TS and SS stenosis after HVLP in a subset of patients with $\mathrm{IIH} .{ }^{3}$ In this paper we present the first report of an IIH patient with immediate post-HVLP TS and SS trans-stenosis pressure gradient reduction and an attendant increase in TS and SS cross-sectional area confirmed using IVUS imaging. Recurrence of the patient's TS-SS stenosis coincided with elevated HVLP opening pressure, and venous sinus stent placement resulted in clinical improvement. Although this report may suggest venous sinus stenosis is a downstream effect of elevated ICP in patients with IIH, we hypothesize that endovascular stenting may obliterate a positive feedback loop involving trans-stenosis pressure gradients, and still benefit appropriately selected patients.

\section{References}

1. De Simone R, Ranieri A, Bonavita V: Starling resistors, autoregulation of cerebral perfusion and the pathogenesis of idiopathic intracranial hypertension. Panminerva Med 59:76-89, 2017

2. Dinkin MJ, Patsalides A: Venous sinus stenting in idiopathic intracranial hypertension: results of a prospective trial. J Neuroophthalmol [epub ahead of print], 2016

3. Horev A, Hallevy H, Plakht Y, Shorer Z, Wirguin I, Shelef I: Changes in cerebral venous sinuses diameter after lumbar puncture in idiopathic intracranial hypertension: a prospective MRI study. J Neuroimaging 23:375-378, 2013

4. Liu KC, Starke RM, Durst CR, Wang TR, Ding D, Crowley RW, et al: Venous sinus stenting for reduction of intracranial pressure in IIH: a prospective pilot study. J Neurosurg [epub ahead of print December 23, 2016. DOI: 10.3171/ 2016.8.JNS16879]

5. Mollan SP, Markey KA, Benzimra JD, Jacks A, Matthews TD, Burdon MA, et al: A practical approach to, diagnosis, assessment and management of idiopathic intracranial hypertension. Pract Neurol 14:380-390, 2014

6. Raper DM, Buell TJ, Ding D, Pomeraniec IJ, Crowley RW, Liu KC: A pilot study and novel angiographic classification for superior sagittal sinus stenting in patients with nonthrombotic intracranial venous occlusive disease. J Neurointerv Surg [epub ahead of print], 2017

7. Raper DM, Ding D, Chen CJ, Buell TJ, Crowley RW, Liu KC: Patency of the vein of Labbé after venous stenting of the transverse and sigmoid sinuses. J Neurointerv Surg [epub ahead of print], 2017

8. Starke RM, Wang T, Ding D, Durst CR, Crowley RW, Chalouhi N, et al: Endovascular treatment of venous sinus stenosis in idiopathic intracranial hypertension: complications, neurological outcomes, and radiographic results. Sci World J 2015:140408, 2015

\section{Disclosures}

The authors report no conflict of interest concerning the materials or methods used in this study or the findings specified in this paper.

\section{Author Contributions}

Conception and design: Buell, Liu. Acquisition of data: Buell, Raper, Pomeraniec, Ding, Liu. Analysis and interpretation of data: Buell, Liu. Drafting the article: Buell. Critically revising the article: all authors. Reviewed submitted version of manuscript: all authors. Approved the final version of the manuscript on behalf of all authors: Buell.

\section{Correspondence}

Thomas J. Buell, Department of Neurosurgery, University of Virginia Health System, Box 800212, Charlottesville, VA 22908. email: tjb4p@hscmail.mcc.virginia.edu. 